\title{
Higher moments of net-kaon multiplicity distributions at RHIC energies for the search of QCD critical point
}

\author{
Amal Sarkar (for the STAR collaboration)* \\ Indian Institute of Technology Bombay \\ Mumbai, India-400076 \\ E-mail: amalercf.rhic.bnl.gov
}

In this paper we report the measurements of the various moments mean $(M)$, standard deviation $(\sigma)$, skewness (S) and kurtosis $(\kappa)$ of the net-kaon multiplicity distribution from Au+Au collisions at $\sqrt{s_{N N}}=7.7$ to $200 \mathrm{GeV}$ in the STAR experiment at RHIC in an effort to locate the critical point in the QCD phase diagram. These moments and their products are related to the thermodynamic susceptibilities of conserved quantities such as net baryon number, net charge, and net strangeness as also to the correlation length of the system. A non-monotonic behavior of these variable will indicate the presence of the critical point. In this work we also present the moments products S $\sigma$, $\kappa \sigma^{2}$ of net-kaon multiplicity distribution as a function of collision centrality and energies. The results are compared with simulation from the AMPT model which do not include the critical point.

8th International Workshop on Critical Point and Onset of Deconfinement

March 11-15

Napa, California, USA

${ }^{*}$ Speaker. 


\section{Introduction}

One of the fundamental goals of the heavy-ion collision experiment is to map the QCD phase diagram as a function of temperature and baryon-chemical potential. Many QCD models based, admit the existence of a critical point which is the quest of present investigations at the Relativistic Heavy Ion Collider (RHIC) at BNL. The correlation length and the magnitude of the fluctuations diverge at the critical point [1,2]. Finite size and time effects in heavy-ion collisions put constraints on the values of $\xi$. However, heavy-ion collision brings down $\xi$ in the range of 2-3 fm from infinity $[2,4]$. It has been shown that the higher non Gaussian moments ( as they depend on higher powers of $\xi$ ) of fluctuations of conserved quantities are much more sensitive to correlation length than variances ( which vary only as $\xi^{2}$ ) [5-8]. For example, the skewness and kurtosis have been shown to be related to the $\xi^{4.5}$ and $\xi^{7}$, respectively [5-9].

In this work, we report the first measurement of the moments of the net-kaon multiplicity distributions as a function of baryon chemical potential which was varied from 410 to $20 \mathrm{MeV}$ by changing the $\sqrt{s_{N N}}=7.7,11.5,19.6,27,39,62.4$ and $200 \mathrm{GeV}$ in $\mathrm{Au}+\mathrm{Au}$ collisions [10]. The data were taken by the STAR experiment in 2010 and 2011 as a part of the beam energy scan (BES) program at RHIC [10]. Different combination of these moment product such as $\mathrm{S} \sigma$ and $\kappa \sigma^{2}$ have been calculated ( to cancel out the dependence on the volume ) as collision centrality[5,9]. Near the critical point these moments products are expected to show a non-monotonic behavior.

\section{Analysis Details}

The STAR(Solenoidal Tracker At RHIC) experiment at Brookhaven National Laboratory provides excellent particle identification and large uniform acceptance at midrapidity within the collision energy range $\sqrt{s_{N N}}=7-200 \mathrm{GeV}$ [10-12]. Ionization energy loss $d E / d x$ of charged particles in the Time Projection Chamber (TPC) was used to identify charged kaons by comparing it to the theoretical (parameterized) expectation along with a $m^{2}$ cut from the information of Time-of-Flight (TOF) detector [11,12]. The analysis was carried out with the event-by-event identified kaons in full azimuthal coverage, within the pseudo-rapidity range $|\eta|<0.5$ and within $0.2<p(\mathrm{GeV} / \mathrm{c})<1.6$. For centrality selection we have used uncorrected charged particle multiplicity within $0.5<|\eta|<1.0$, to avoid the autocorrelation[13]. For each centrality, the average numbers of participants $\left(\left\langle N_{\text {part }}\right\rangle\right)$ are obtained by Glauber model calculations. The results are corrected for the finite centrality bin width effects[13].

\section{Results}

The raw net-kaon $\left(\Delta N_{K}=N_{K^{+}}-N_{K^{-}}\right)$multiplicity distribution in Au+Au collisions at $\sqrt{s_{N N}}=$ $7.7-200 \mathrm{GeV}$ for various collision centralities at midrapidity $(|\eta|<0.5)$, are shown in the Figure 1. The mean of net-kaon $\left(\Delta N_{K}\right)$ distribution shifts towards zero from low to high energies.

The four moments ( $M, \sigma, \mathrm{S}$, and $\kappa$ ) of the $\Delta N_{K}$ distributions at various collision energies are plotted as a function of average number of participants $\left\langle N_{\text {part }}\right\rangle$. The fitted doted lines are the expected values from the central limit theorem (CLT), which varies as volume's $x, \sqrt{x}, \frac{1}{\sqrt{x}}$ and $\frac{1}{x}$ respectively. Error bars are statistical only, calculated from Delta theorem [14]. 

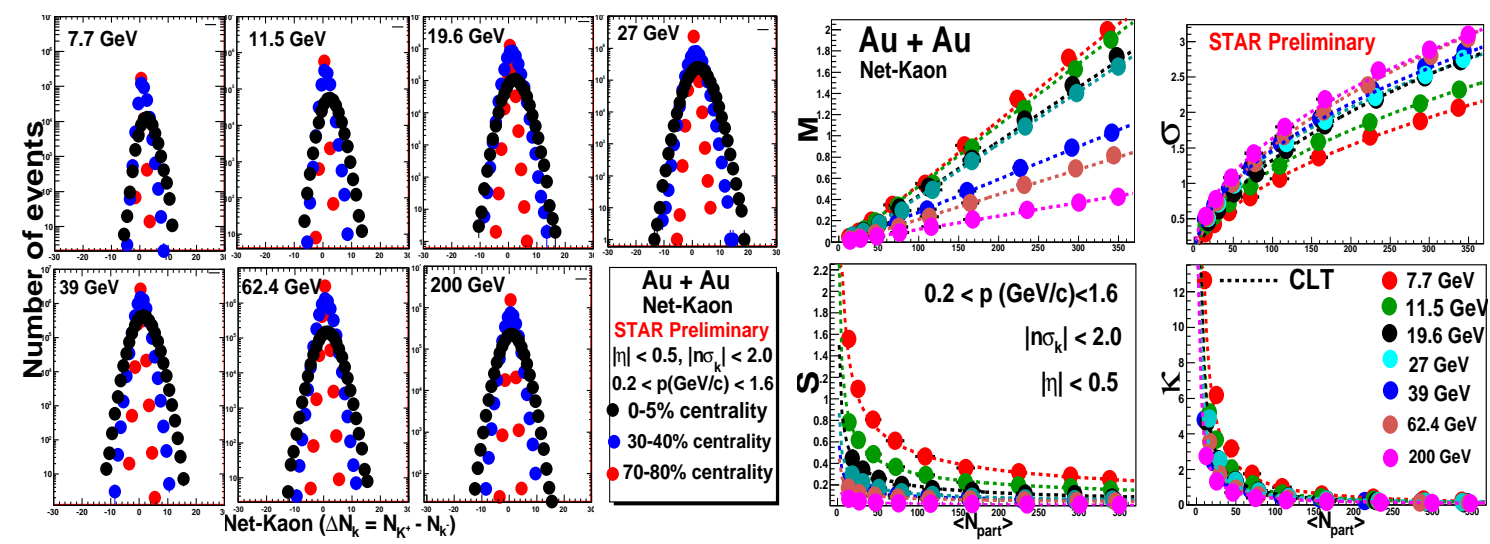

Figure 1: $\Delta N_{K}$ multiplicity distribution in Au+Au collisions at $\sqrt{s_{N N}}=7.7-200 \mathrm{GeV}$ for various collision centralities at mid-rapidity $(|\eta|<0.5)$ and the moments calculated from these distribution after applying the bin-width correction[13]

The centrality dependence $S \sigma$ and $\kappa \sigma^{2}$ are shown in Figure 2 compared with the baseline from Poisson statistics for the seven available beam energies. The Poisson baseline has been calculated from the mean value of the $N_{K^{+}}$and $N_{K^{-}}$distribution. Figure. 2 shows $\mathrm{S} \sigma$ value is independent of centrality within $15 \%$. S $\sigma$ value is greater than the Poisson expectation for beam energy below 200 $\mathrm{GeV}$ and $\mathrm{S} \sigma$ increases with decreasing collision energies. Within the statistical uncertainty $\kappa \sigma^{2}$ does not show a strong centrality dependence.
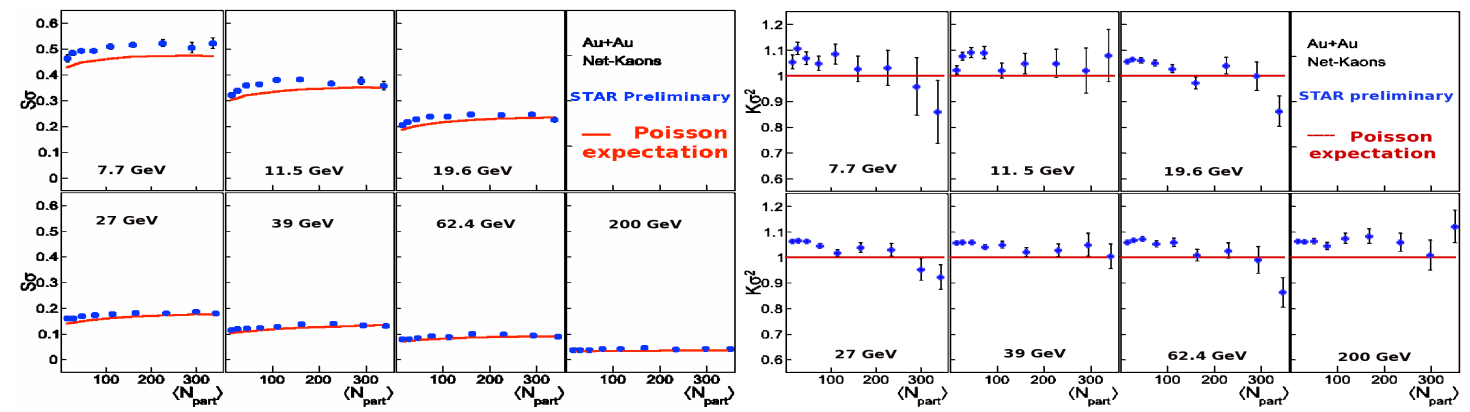

Figure 2: Centrality dependance of $\mathrm{S} \sigma$ and $\kappa \sigma^{2}$

In Figure 3 the energy dependency of the moment product $S \sigma$ and $\kappa \sigma^{2}$ for the top central 0-5\% Au+Au collisions is shown. The data has been compared with the Poisson baseline and with the AMPT(default) model result. Within the statistical uncertainty AMPT calculations agree well with the data.

\section{Summary}

The first measurements of the higher moments kurtosis $(\kappa)$, skewness $(\mathrm{S})$, and variance $\left(\sigma^{2}\right)$ of 

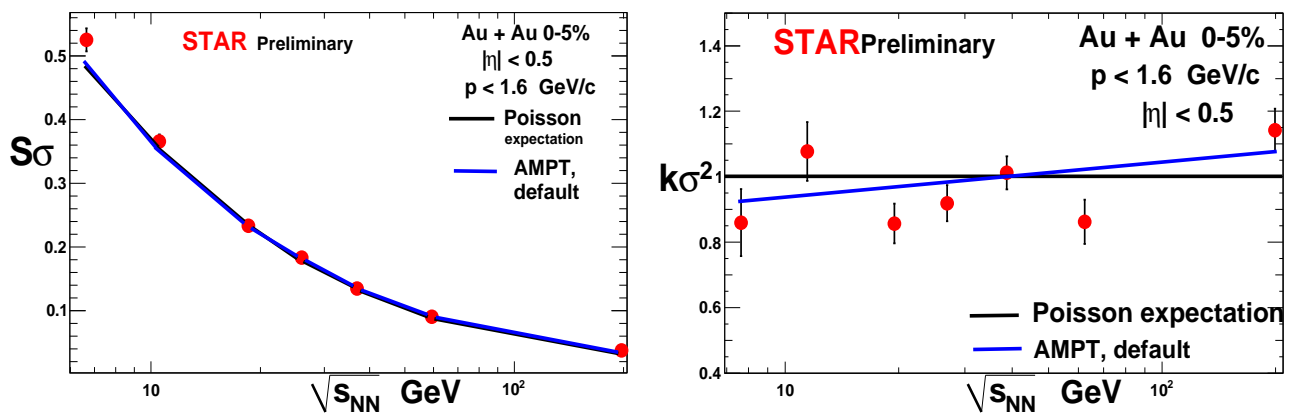

Figure 3: Energy dependence of $\mathrm{S} \sigma$ and $\mathrm{k} \sigma^{2}$

net-kaon multiplicity $\left(\Delta N_{K}=N_{K^{+}}-N_{K^{-}}\right)$distributions at mid-rapidity $(|\eta|<0.5)$ in $0.2<p<1.6$ $\mathrm{GeV} / \mathrm{c}$ for $\mathrm{Au}+\mathrm{Au}$ collisions at $\sqrt{s_{N N}}=7.7,11.5,19.6,62.4$, and $200 \mathrm{GeV}$ corresponding to baryon chemical potentials $\left(\mu_{B}\right)$ between 400 and $20 \mathrm{MeV}$ were shown. The centrality dependence of moments follows the central limit theorem well. New observables $S \sigma$ and $\kappa \sigma^{2}$ derived from the $\Delta N_{K}$ distribution to search for the critical point in heavy-ion collisions are discussed. S $\sigma$ values are found to be independent of centrality as a function of $\left\langle N_{\text {part }}\right\rangle$ for all available collision energies. $\kappa \sigma^{2}$ values are also found to be constant within the statistical uncertainties as a function of $\left\langle N_{\text {part }}\right\rangle$ for all collision energies studied. $\mathrm{S} \sigma$ and $\kappa \sigma^{2}$ are consistent with expectations from AMPT model without the critical point in top central 0-5\% Au+Au collisions. No significant enhancement of moment products was observed compared to the Poisson baseline at presently available energies.

\section{References}

[1] Y. Aoki et al., arXiv:1007.2613

[2] M. M. Aggarwal et al., arXiv:1007.2613

[3] B. Berdnikov et al., Phys. Rev. D 61, 105017 (2000).

[4] M. Cheng et al., Phys. Rev. D 79, 074505 (2009).

[5] STAR Collaboration, Phys. Rev. Lett 105, 022302 (2010).

[6] M. A. Stephanov, Phys. Rev. Lett. 102, 032301 (2009).

[7] M. A. Stephanov, Phys. Rev. Lett. 107, 052301(2011).

[8] C. Athanasiou et al., Phys. Rev. D 82, 074008 (2010).

[9] F. Karsch et al., Phys. Lett. B 695, pp 136-142 (2011).

[10] STAR Collaboration, Phys. Rev. C 81, 024911 (2010).

[11] M. Shao et al., Nucl.Instrum.Meth. A 558, pp 419-429 (2006).

[12] STAR Collaboration, Nuclear Physics A 774, pp 956-958 (2006).

[13] X. Luo, J. Phys. Conf. Ser. 316, 445012003 (2011).

[14] X. Luo, J. Phys. G: Nucl. part. Phys 39, 025008 (2012). 